Article

\title{
Synthesis of Novel $\beta$-Keto-Enol Derivatives Tethered Pyrazole, Pyridine and Furan as New Potential Antifungal and Anti-Breast Cancer Agents
}

\author{
Smaail Radi ${ }^{1, *}$, Said Tighadouini ${ }^{1}$, Olivier Feron ${ }^{2}$, Olivier Riant ${ }^{3}$, Mohammed Bouakka ${ }^{4}$, \\ Redouane Benabbes ${ }^{4}$ and Yahia N. Mabkhot ${ }^{5}$ \\ Received: 4 September 2015 ; Accepted: 2 November 2015 ; Published: 10 November 2015 \\ Academic Editor: Richard A. Bunce \\ 1 Department of Chemistry, Faculty of Sciences, University Mohamed I, Oujda-60000, Morocco; \\ tighadouinis@gmail.com \\ 2 Angiogenesis and Cancer Research Lab, Pole of Pharmacology and Therapeutics-FATH5349, \\ Institute of Experimental and Clinical Research, Université catholique de Louvain (UCL), Brussels 1200, \\ Belgium; olivier.feron@uclouvain.be \\ 3 Molecules, Solids and Reactivity (MOST), Institute of Condensed Mater and Nanosciences (IMCN), \\ Université catholique de Louvain (UCL), Place Louis Pasteur 1, Louvain-la-Neuve 1348, Belgium; \\ olivier.riant@uclouvain.be \\ 4 Department of Biologie, Faculty of Sciences, University Mohamed I, Oujda-60000, Morocco; \\ bouakkam@yahoo.fr (M.B.); red.bes72@gmail.com (R.B.) \\ 5 Department of Chemistry, Faculty of Science, King Saud University, P. O. Box 2455, Riyadh 11451, \\ Saudi Arabia; yahia@ksu.edu.sa \\ * Correspondence: radi_smaail@yahoo.fr; Tel.: +212-536-500-601; Fax: +212-536-500-603
}

\begin{abstract}
Recently, a new generation of highly promising inhibitors bearing $\beta$-keto-enol functionality has emerged. Reported herein is the first synthesis and use of novel designed drugs based on the $\beta$-keto-enol group embedded with heterocyclic moieties such as pyrazole, pyridine, and furan, prepared in a one-step procedure by mixed Claisen condensation. All the newly synthesized compounds were characterized by FT-IR, ${ }^{1} \mathrm{H}-\mathrm{NMR},{ }^{13} \mathrm{C}-\mathrm{NMR}, \mathrm{ESI} / \mathrm{LC}-\mathrm{MS}$, elemental analysis, and evaluated for their in vitro antiproliferative activity against breast cancer (MDA-MB241) human cell lines and fungal strains (Fusarium oxysporum f.sp albedinis FAO). Three of the synthesized compounds showed potent activity against fungal strains with $\mathrm{IC}_{50}$ values in the range of $0.055-0.092 \mu \mathrm{M}$. The results revealed that these compounds showed better $\mathrm{IC}_{50}$ values while compared with positive controls.
\end{abstract}

Keywords: keto-enols; heterocycles; breast cancer; fungal strains

\section{Introduction}

Heterocyclic compounds with $\beta$-keto-enol moieties are well established as important, biologically effective compounds. Their versatile utility in the world of medicinal chemistry is firmly established [1,2]. Among this class of drug, we cite anti-HIV drugs S-1360 (Shionogi, Ltd., Florham Park, NJ, USA) [3] and L-708,906 (Merck Research Laboratories, Boston, MA, USA) [4] in clinical trials as well as P13 $\left(\mathrm{IC}_{50}=1.1 \mu \mathrm{M}\right)$ [5], the 5-CITEP designed by the National Cancer Institute (Bethesda, $\mathrm{MD}, \mathrm{USA})$ [3], and $\mathrm{AIV}\left(\mathrm{IC}_{50}=0.3 \mu \mathrm{M}\right)$, an inhibitor of anti-influenza virus [6] (Figure 1), etc.

In this context, we note also the interest in curcumin [7] derivatives with $\beta$-keto-enol pharmacophore sites (Figure 1) which have spurred numerous studies in medicinal chemistry owing to their capability of eliciting antioxidant [8], anti-HIV [9], antitumor [10-12], and anti-inflammatory [13] activities. 
<smiles>O=C(/C=C(\O)c1nc[nH]n1)c1ccc(Cc2ccc(F)cc2)o1</smiles>

S-1360<smiles>O=C(/C=C(\O)c1nn[nH]n1)c1c[nH]c2ccc(Cl)cc12</smiles>

5-CITEP<smiles>O=C(O)/C=C(\O)C(=O)c1c[nH]c2ccccc12</smiles>

P13<smiles>[R]N1CCC([R])(C(=O)C=C(O)C(=O)O)CC1</smiles>

AIV<smiles>CCCCOc1cc(OC(=O)OCc2ccccc2)cc(C(=O)C=C(O)C(=O)O)c1</smiles>

L-708,906<smiles>COc1ccc(/C=C/C(=O)/C=C(O)/C=C/c2ccc(OC)c(O)c2)cc1O</smiles>

Curcumin

Figure 1. Representative drugs containing keto-enol functionality.

The $\beta$-keto-enol pharmacophore site of these hybrid drug molecules has the potential advantage of being active against all genotypes of the virus and drug-resistant variants. Multistage processes may explain the biological responses of this motif ( $\beta$-keto-enol), such as (i) its penetration into blood vessel walls and plasma cell membranes; (ii) its interaction with the active site; (iii) its ability to chelate with metals in biological processes; and (iv) its reaction with oxygen (under aerobic conditions) or with cell macromolecules (in hypoxic conditions) resulting in oxidative stress, the modulation of gene expression, and a complex immune response to hapten-conjugate adducts.

Consequently, significant effort is devoted to the search for drug-like scaffolds bearing the $\beta$-keto-enol pharmacophore. Recently, several molecules were designed, such as the calix[4]arene derivatives, containing the triazolyl keto-enol moiety showing potent integrase strand transfer inhibitory activity [14], keto-enol tetrazoles, and triazoles as anti-HCV agents [15], and coumarinyl chalcones, exhibiting high selectivity for the breast cancer cell lines [16].

Accordingly, described herein is the first synthesis and examination of some hybrid drug molecules bearing the $\beta$-keto-enol functionality as a useful motif, especially in fungal and cancer activities.

\section{Results and Discussion}

\subsection{Chemistry}

The target compounds based on $\beta$-keto-enol group-tethered pyrazole, pyridine, and furan were prepared by a one-pot in situ condensation as illustrated and outlined in Schemes 1 and 2.

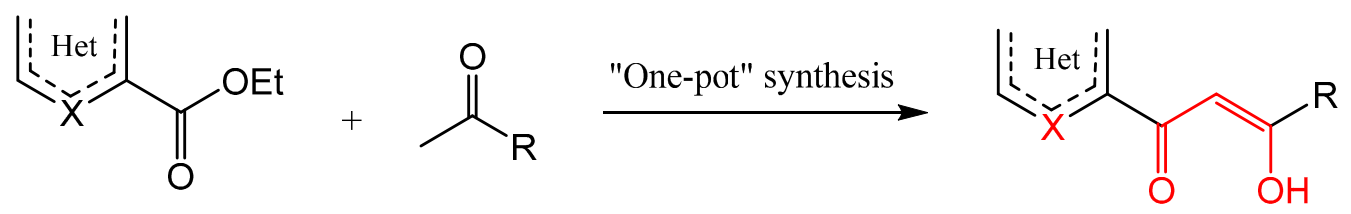

Het $=$ Pyrazole, Pyridine, Furan $\mathrm{R}=\mathrm{CH}_{3}, \mathrm{C}_{6} \mathrm{H}_{5}, p-\mathrm{Me}-\mathrm{C}_{6} \mathrm{H}_{4}, p-\mathrm{OMe}-\mathrm{C}_{6} \mathrm{H}_{4}$

Scheme 1. Reagents and conditions: $\mathrm{Na}$, Toluene, rt, two days, then acetic acid.

The sodium metal-mediated condensation of ketone derivatives with ethyl heterocycle-2-carboxylates exclusively afforded the target products in their enol tautomeric form. The reaction, as a mixed Claisen condensation, was carried out under mild conditions (room 
temperature, two days), using toluene as a solvent and sodium metal as the base. The reaction was slow and gave better results (with respect to the purity of the compounds).

The synthesis began with the formation of a ketone enolate nucleophile in cool conditions $\left(0^{\circ} \mathrm{C}\right)$. After adding the appropriate heterocyclic carboxylate, the resulting mixture was stirred at room temperature for two days. The formed enolate initially underwent nucleophilic attack at the ester carbonyl to produce tetrahedral intermediate (A).

The expulsion of the ethoxide ion from the unstable tetrahedral intermediate of the initial Claisen adduct yielded a $\beta$-diketone $(\mathbf{B})$. The expelled base $\left(\mathrm{EtO}^{-} \mathrm{Na}^{+}\right)$then removed an acidic alpha proton from the $\beta$-diketone to generate a stabilized enolate ion product $(\mathbf{C})$ as a precipitated salt. This formed precipitate was filtered, washed with toluene, dissolved in water, and neutralized with acetic acid to $\mathrm{pH}=5$ to afford the title products in acceptable yield after being filtered through silica using $\mathrm{CH}_{2} \mathrm{Cl}_{2} / \mathrm{MeOH}$ as an eluant. The mechanism for the formation of these target products is given in Scheme 2 .

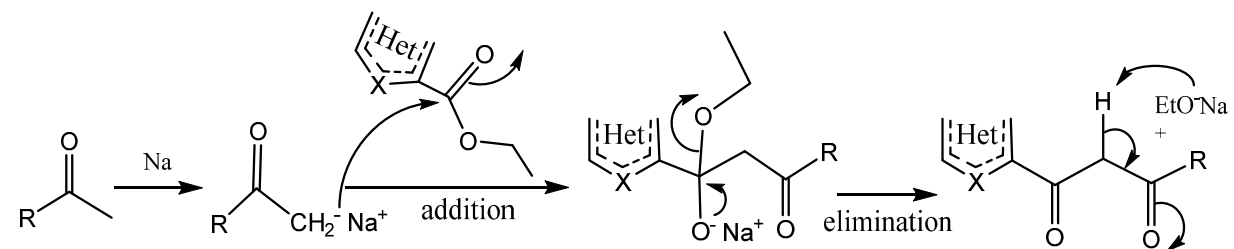

(A)

(B)

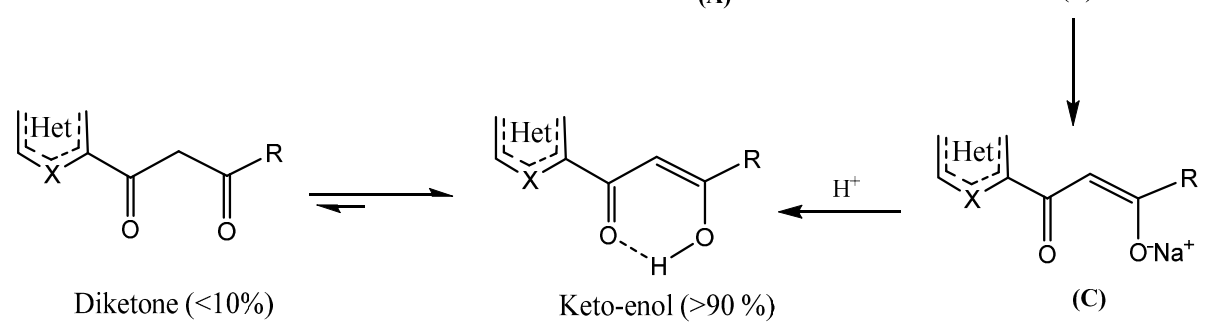

Scheme 2. Proposed mechanism of the formed products.

It is emphasized that our products exist exclusively in "3-hydroxy-alk-2-en-1-one" form as confirmed by the spectral data; these tautomeric forms were also confirmed by XRD and the results will appear in due course [17]. However, two products (similar to 5 and 6) were obtained in previous work by others, in the "4-hydroxy-alk-3-en-2-one" tautomeric form under different conditions ( $\mathrm{NaH}, \mathrm{Et}_{2} \mathrm{O}$, reflux) [18].

The $\beta$-keto-enol form is greatly favored over the $\beta$-diketone form because of the conjugation of the enol with the carbonyl group, and the stability gained, by a strong six-centered intramolecular hydrogen bond. The $\beta$-keto-enol interconversion rate $(>90 \%)$ was determined using the ${ }^{1} \mathrm{H}-\mathrm{NMR}$ integration of signals from the enol $=\mathrm{C}-\mathrm{H}$ and the ketone $\mathrm{CH}_{2}$. Indeed, according to the NMR spectra, the parent $\beta$-diketones exist almost exclusively in the enol form and only a trace of the keto form is seen around $4 \mathrm{ppm}$. In DEPT-135, a very small negative signal from $\mathrm{CH}_{2}$ was also observed. Finally, crystals of most of the $\beta$-keto-enols were isolated from methanol by slow evaporation. Compound 6 was recently reported in a different way [19].

\subsection{Biological Activities}

All synthesized $\beta$-keto-enol heterocycles were evaluated for their activity against breast cancer (MDA-MB241) human cell lines using normoxic conditions [20], and against fungal strains (Fusarium oxysporum f.sp albedinis FAO) using the agar diffusion technique (ADT) [21]. It is of note that all products were also tested against three bacterial strains (Echerichia coli, Bacillus subtilis, and Micrococcus luteus), but no significant effect was observed against these organisms. 
Table 1. Breast cancer and fungal inhibitory activities of synthesized heterocycle $\beta$-keto-enols.<smiles>[R]/C(O)=C/C(=O)C(=C)/[X]([H])=C/C=C</smiles>

General Structure

\begin{tabular}{|c|c|c|c|c|c|c|}
\hline \multicolumn{3}{|c|}{ Products } & \multicolumn{2}{|c|}{ MDA-MB241 } & \multicolumn{2}{|c|}{ Fusarium Oxysporum f.sp Albedinis } \\
\hline No. & Heterocycles & $\mathbf{R}$ & $\mathrm{IC}_{50}(\mu \mathrm{g} / \mathrm{mL})$ & $\mathrm{IC}_{50}(\mu \mathrm{M})$ & $\mathrm{IC}_{50}(\mu \mathrm{g} / \mathrm{mL})$ & $\mathrm{IC}_{50}(\mu \mathrm{M})$ \\
\hline 1 & & $\mathrm{CH}_{3}$ & 46.20 & 256.38 & 0.01 & 0.055 \\
\hline 2 & & $\mathrm{C}_{6} \mathrm{H}_{5}$ & 44.33 & 183.00 & 12.83 & 53.39 \\
\hline 3 & & $p-\mathrm{Me}-\mathrm{C}_{6} \mathrm{H}_{4}$ & 21.95 & 85.65 & 142 & 554.03 \\
\hline 4 & & $p-\mathrm{MeO}-\mathrm{C}_{6} \mathrm{H}_{4}$ & 34.93 & 128.30 & 150 & 550.86 \\
\hline 5 & & $\mathrm{CH}_{3}$ & 47.00 & 288.04 & 0.013 & 0.079 \\
\hline 6 & & $\mathrm{C}_{6} \mathrm{H}_{5}$ & 17.62 & 78.23 & 16.43 & 72.94 \\
\hline 7 & & $p-\mathrm{Me}-\mathrm{C}_{6} \mathrm{H}_{4}$ & 128.67 & 537.8 & 35.80 & 149.62 \\
\hline 8 & & $p-\mathrm{MeO}-\mathrm{C}_{6} \mathrm{H}_{4}$ & 28.97 & 113.50 & $\mathrm{~N} / \mathrm{A} *$ & $\mathrm{~N} / \mathrm{A} *$ \\
\hline 9 & & $\mathrm{CH}_{3}$ & $\mathrm{~N} / \mathrm{A}^{*}$ & $\mathrm{~N} / \mathrm{A}^{*}$ & 0.014 & 0.092 \\
\hline 10 & & $\mathrm{C}_{6} \mathrm{H}_{5}$ & 18.79 & 87.72 & 68.45 & 319.53 \\
\hline
\end{tabular}

${ }^{*} \mathrm{~N} / \mathrm{A}$ means non applicable because $\mathrm{IC}_{50}>100 \mu \mathrm{g} / \mathrm{mL}$. 
However, the results of the anticancer and antifungal effects were very significant and are given in Table 1, respectively. Most of these molecules were cytotoxic against breast cancer cell lines in a dose-dependent manner. The activity followed the structure activity relationships (SARs) and showed an interesting dependence on the substitution pattern. Considering the influence of substituent $R$, it was found that the phenyl residue leads to a stronger growth inhibition [18]. This was especially evident for products 6 and 10 . The concentration required to induce the activity $\left(\mathrm{IC}_{50}\right)$ was more pronounced for compounds 3,6 and 10 with $\mathrm{IC}_{50}$ values of $21.95,17.62$, and $18.79 \mu \mathrm{g} / \mathrm{mL}$, respectively. Beside this observation, we also noted the effect of the heterocycle groups for appreciable biological activity.

These structures have also led to unexpected antifungal activity. Indeed, compounds 1, 5, and 9 with methyl in the $\mathrm{R}$ position had the most potent activity with $\mathrm{IC}_{50}$ values of $0.055,0.079$, and $0.092 \mu \mathrm{M}$, respectively. This result was better than all the described products. We noted that the substitution of methyl in the $\mathrm{R}$ position was essential for this biological activity. The aryl groups strongly decreased the activity. This suggests that the marked bioactivity of the heterocyclic compounds was sensitive to modifications and could be further exploited to determine the structure activity relationship around this novel class of fungal inhibitors. Other structural modifications to these active compounds as antifungal and anti-HIV candidates are currently in progress.

\section{Experimental}

\subsection{General Information}

All commercial reagents were analytical grade (Aldrich, purity $>99 \%$, St. Louis, MO, USA). Melting points were measured using a BUCHÏ 510 m.p. apparatus (Oujda, Morocco). ${ }^{1} \mathrm{H}-$ and ${ }^{13}$ C-NMR spectra were performed on a Bruker AC 300 spectrometer (CNRS, Rabat, Morocco) (300 MHz for ${ }^{1} \mathrm{H}$ and $75.47 \mathrm{MHz}$ for ${ }^{13} \mathrm{C}$ spectra). JEOL JMS DX-300 mass spectrometer (Rabat, Morocco) was used for the determination of molecular weights. Infrared (IR) spectra were recorded on a Shimadzu infrared spectrophotometer (Oujda, Morocco) using the $\mathrm{KBr}$ disc technique. The formazan obtained at the end of the experiment in MTT assays was measured by means of a Perkin Elmer Victor X4 Microplate reader (Brussels, Belgium).

\subsection{General Procedure for the Synthesis of $\beta$-Keto-Enol Heterocycles}

To a suspension of sodium $(15.21 \mathrm{mmol})$ in $20 \mathrm{~mL}$ of toluene, the appropriate heterocyclic carboxylate (12.01 mmol) in $25 \mathrm{~mL}$ of toluene was slowly added; then acetone or aryl methyl ketones $(12.01 \mathrm{mmol})$ in $10 \mathrm{~mL}$ of toluene was added at $0{ }^{\circ} \mathrm{C}$. The resulting mixture was stirred at room temperature for two days. The precipitate formed was filtered, washed with toluene, dissolved in water, and neutralized with acetic acid to $\mathrm{pH} 5$. After extraction with $\mathrm{CH}_{2} \mathrm{Cl}_{2}$, the organic layer was dried over anhydrous sodium sulfate and concentrated in vacuo. The obtained residue was filtered through silica using $\mathrm{CH}_{2} \mathrm{Cl}_{2} / \mathrm{MeOH}$ as eluant to give the desired products 1-10 as a white solid in $35 \%-48 \%$ yield. $\beta$-keto-enol forms were recrystallized from methanol (95\%) to obtain target compounds $\mathbf{1 - 1 0}$ which were confirmed by FT-IR, ${ }^{1} \mathrm{H}-\mathrm{NMR},{ }^{13} \mathrm{C}-\mathrm{NMR}$, elemental analysis, and mass spectroscopy.

(Z)-1-(1.5-Dimethyl-1H-pyrazol-3-yl)-3-hydroxybut-2-en-1-one (1). Yellow crystal; yield: 35\%; m.p. 83-84 ${ }^{\circ} \mathrm{C} ; R_{f}=0.66$ (diethyl ether)/silica. IR $\left(\mathrm{KBr}, \mathrm{cm}^{-1}\right): v(\mathrm{OH})=3422 ; v(\mathrm{C}=\mathrm{O})=1619 ; v$ $($ enolic $\mathrm{C}=\mathrm{C})=1509 ;{ }^{1} \mathrm{H}-\mathrm{NMR}\left(\mathrm{CDCl}_{3}\right): \delta 2.11\left(\mathrm{~s}, 3 \mathrm{H},-\mathrm{CH}_{3}\right) ; 2.27\left(\mathrm{~s}, 3 \mathrm{H}, \mathrm{Pz}_{-}-\mathrm{CH}_{3}\right) ; 3.83\left(\mathrm{~s}, 3 \mathrm{H}, \mathrm{N}-\mathrm{CH}_{3}\right)$;

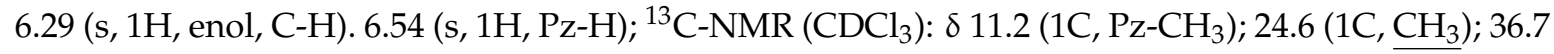

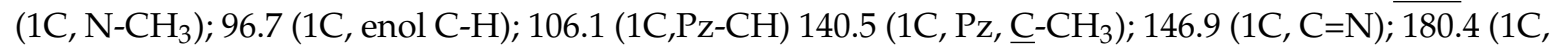
$\mathrm{C}=\mathrm{O}) ; 190.0(\mathrm{C}-\mathrm{OH}) ; \mathrm{MS}: m / z, 181.00(\mathrm{M}+\mathrm{H})^{+}$. Anal. Cald. for C_9H_12N_2O_2: C, 59.99; H, 6.71; $\mathrm{N}, 15.55$. Found: $\mathrm{C}, 60.11 ; \mathrm{H}, 6.83 ; \mathrm{N}, 15.43$. 
(Z)-1-(1,5-Dimethyl-1H-pyrazol-3-yl)-3-hydroxy-3-phenylprop-2-en-1-one (2). Yellow crystal; yield: 32\%; m.p. $108-110^{\circ} \mathrm{C} ; R_{f}: 0.88\left(\mathrm{CH}_{2} \mathrm{Cl}_{2} / \mathrm{MeOH} 9 / 1 /\right.$ silica $), \mathrm{IR}\left(\mathrm{KBr}, \mathrm{cm}^{-1}\right): v(\mathrm{OH})=3416 ; v(\mathrm{C}=\mathrm{O})=1639$; $v($ enolic $\mathrm{C}=\mathrm{C})=1518 ;{ }^{1} \mathrm{H}-\mathrm{NMR}\left(\mathrm{DMSO}_{-} d_{6}\right): \delta 2.29\left(\mathrm{~s}, 3 \mathrm{H}, \mathrm{Pz}-\mathrm{CH}_{3}\right) ; 3.83\left(\mathrm{~s}, 3 \mathrm{H}, \mathrm{N}-\mathrm{CH}_{3}\right) ; 6.60(\mathrm{~s}, 1 \mathrm{H}$, enol, C-H); 7.02 (s, 1H, Pz-H); 7.58 (m, 3H, Ar-H $\left.\mathrm{H}_{4,3,5}\right) ; 7.95\left(\mathrm{~m}, 2 \mathrm{H}, \mathrm{Ar}-\mathrm{H}_{2,6}\right) .{ }^{13} \mathrm{C}-\mathrm{NMR}\left(\mathrm{DMSO}-d_{6}\right): \delta$ 11.9 (1C, $\left.\mathrm{Pz}_{-} \mathrm{CH}_{3}\right) ; 37.3$ (1C, $\mathrm{CH}_{3}-\mathrm{N}$ ); 93.3 (1C, enol C-H); 106.1 (1C, $\left.\underline{\mathrm{CH}}-\mathrm{Pz}\right) ; 127.3$ (2C, Ar-C 2,6$) ; 128.8$ (1C, Ar- $\left.\mathrm{C}_{4}\right) ; 129.3$ (2C, Ar- $\left.\mathrm{C}_{3,5}\right) ; 148.8\left(1 \mathrm{C}, \mathrm{Pz}, \underline{\mathrm{C}}-\mathrm{CH}_{3}\right) ; 181.7$ (1C, $\left.\underline{\mathrm{C}}-\mathrm{OH}\right) ; 183.5$ (1C, $\left.\underline{\mathrm{C}}=\mathrm{O}\right) ; \mathrm{MS}: \mathrm{m} / z$, $243.10(\mathrm{M}+\mathrm{H})^{+}$.

(Z)-1-(1,5-Dimethyl-1H-pyrazol-3-yl)-3-hydroxy-3-p-tolyprop-2-en-1-one (3). Yellow crystal; yield: 22\%; m.p. $132-134{ }^{\circ} \mathrm{C} ; R_{f}: 0.29$ (diethyl ether)/silica. IR $\left(\mathrm{KBr}, \mathrm{cm}^{-1}\right): v(\mathrm{OH})=3412 ; v(\mathrm{C}=\mathrm{O})=1642$; $v($ enolic $\mathrm{C}=\mathrm{C})=1536 ;{ }^{1} \mathrm{H}-\mathrm{NMR}\left(\mathrm{DMSO}-d_{6}\right): \delta 2.26\left(\mathrm{~s}, 3 \mathrm{H}, p-\mathrm{CH}_{3}-\mathrm{Ar}\right) ; 2.36\left(\mathrm{~s}, 3 \mathrm{H}, \mathrm{Pz}-\mathrm{CH}_{3}\right) ; 3.82$ (s, 3H, CH $3-\mathrm{N}) ; 6.67$ (d, 1H, Pz-H); 6.98 (s, 1H, enol, C-H); 7.32 (m, 2H, Ar- $\left.\mathrm{H}_{3,5}\right) ; 7.85$ (m, 2H, Ar- $\mathrm{H}_{2,6}$ ). ${ }^{13} \mathrm{C}-\mathrm{NMR}\left(\mathrm{DMSO}-d_{6}\right)$ : $\delta 11.2\left(1 \mathrm{C}, \mathrm{Pz}_{\mathrm{C}} \mathrm{CH}_{3}\right) ; 21.6(1 \mathrm{C}, p-\mathrm{CH} 3-\mathrm{Ar}) ; 37.3\left(1 \mathrm{C}, \mathrm{CH}_{3}-\mathrm{N}\right) ; 92.8(1 \mathrm{C}$, enol C-H); 106.0 (1C, $\underline{\mathrm{C}} \mathrm{H}-\mathrm{Pz}) ; 128.1$ (2C, Ar- $\left.\mathrm{C}_{2,6}\right) ; 129.9$ (2C, Ar-C 3,5$) ; 182.2$ (1C, $\left.\underline{\mathrm{C}}-\mathrm{OH}\right) ; 182.9$ (1C, $\left.\underline{\mathrm{C}}=\mathrm{O}\right) ; \mathrm{MS}: \mathrm{m} / z$, $257.11(\mathrm{M}+\mathrm{H})^{+}$.

(Z)-1-(1,5-Dimethyl-1H-pyrazol-3-yl)-3-hydroxy-3-(4-methoxyphenyl)prop-2-en-1-one (4). Yellow crystal; yield: $31 \%$; m.p. $122-124{ }^{\circ} \mathrm{C}$; $R_{f}$ : $0.61\left(\mathrm{CH}_{2} \mathrm{Cl}_{2} / \mathrm{MeOH} 8 / 2\right) /$ silica. IR $\left(\mathrm{KBr}, \mathrm{cm}^{-1}\right): \vee(\mathrm{OH})=3432$; $v(\mathrm{C}=\mathrm{O})=1678 ; v($ enolic $\mathrm{C}=\mathrm{C})=1528 ;{ }^{1} \mathrm{H}-\mathrm{NMR}\left(\mathrm{DMSO}_{6}\right): \delta 2.26\left(\mathrm{~s}, 3 \mathrm{H}, \mathrm{O}-\mathrm{CH}_{3}\right) ; 3.82(\mathrm{~s}, 3 \mathrm{H}$, Pz-CH $\left.{ }_{3}\right) ; 3.83$ (s, 3H, CH $\left.3-\mathrm{N}\right): 6.65$ (s, 1H, Pz-H); 6.94 (s, 1H, enol, C-H); 7.04 (m, 2H, Ar-H 3,5$) ; 7.94$ $\left(\mathrm{m}, 2 \mathrm{H}, \mathrm{Ar}-\mathrm{H}_{2,6}\right) ;{ }^{13} \mathrm{C}-\mathrm{NMR}$ (DMSO-d $\left.d_{6}\right): \delta 11.1\left(1 \mathrm{C}, \mathrm{Pz}^{-\mathrm{CH}_{3}}\right) ; 37.3\left(1 \mathrm{C}, \mathrm{CH}_{3}-\mathrm{N}\right) ; 56.0(1 \mathrm{C}, p$-OCH3-Ar); 92.2 (1C, enol C-H); 106.1 (1C, =ㅡH-Pz); 114.6 (2C, Ar-C $\left.C_{3,5}\right) ; 129.6$ (2C, Ar-C 2,6$) ; 181.7(1 \mathrm{C}, \underline{\mathrm{C}}-\mathrm{OH})$; $182.9(1 \mathrm{C}, \underline{\mathrm{C}}=\mathrm{O})$; MS: $m / z, 273.06(\mathrm{M}+\mathrm{H})^{+}$.

(Z)-3-Hydroxy-1-(pyridin-2-yl)but-2-en-1-one (5). Brown powder; yield: 48\%; m.p. $=59-61{ }^{\circ} \mathrm{C} ; R_{f}: 0.27$ $\left(\mathrm{CH}_{2} \mathrm{Cl}_{2} / \mathrm{MeOH} 9 / 1\right) /$ silica. IR $\left(\mathrm{KBr}, \mathrm{cm}^{-1}\right): v(\mathrm{OH})=3448 ; v(\mathrm{C}=\mathrm{O})=1611 ; v$ (enolic $\left.\mathrm{C}=\mathrm{C}\right)=1565$; ${ }^{1} \mathrm{H}-\mathrm{NMR}\left(\mathrm{CDCl}_{3}\right): \delta 2.23\left(\mathrm{~s}, 3 \mathrm{H},-\mathrm{CH}_{3}\right) ; 6.94(\mathrm{~s}, 1 \mathrm{H}, \mathrm{enol}, \mathrm{C}-\mathrm{H}) ; 7.42\left(\mathrm{t}, 1 \mathrm{H}, \mathrm{Py}-\mathrm{H}_{\beta}\right) ; 7.85\left(\mathrm{t}, 1 \mathrm{H}, \mathrm{Py}-\mathrm{H}_{\gamma}\right)$; $8.08\left(\mathrm{~d}, 1 \mathrm{H}, \mathrm{Py}-\mathrm{H}_{\delta}\right) ; 8.66(\mathrm{~d}, 1 \mathrm{H}, \mathrm{Py}-\mathrm{H} \alpha) ;{ }^{13} \mathrm{C}-\mathrm{NMR}\left(\mathrm{CDCl}_{3}\right): \delta 26.2\left(1 \mathrm{C}, \mathrm{CH}_{3}-\mathrm{C}=\mathrm{O}\right) ; 97.8(1 \mathrm{C}$, enol, $\mathrm{CH}) ; 123.0\left(1 \mathrm{C}, \mathrm{Py}-\mathrm{C}_{\delta}\right) ; 126.4\left(1 \mathrm{C}, \mathrm{Py}-\mathrm{C}_{\beta}\right) ; 138.0$ (1C, Py-C $\left.{ }_{\gamma}\right) ; 148.5$ (1C, Py-C $\left.\alpha\right) ; 151.4\left(1 \mathrm{C}, \mathrm{Py}-\mathrm{C}_{\varepsilon}\right) ;$ $179.3(1 \mathrm{C}, \mathrm{C}-\mathrm{O}), 195.6(1 \mathrm{C}, \mathrm{C}=\mathrm{OH})$; MS: $\mathrm{m} / \mathrm{z}, 164.07(\mathrm{M}+\mathrm{H})^{+}$. Anal. Calcd. for $\mathrm{C}_{9} \mathrm{H}_{9} \mathrm{NO}_{2}: \mathrm{C}, 66.25$, H, 5.56; N, 8.58. Found: C, 66.31; H, 5.62, N, 5.40.

(Z)-3-Hydroxy-3-phenyl-1-(pyridin-2-yl)prop-2-en-1-one (6). Red powder; yield: 32\%; m.p. $=78-80{ }^{\circ} \mathrm{C} ; \quad R_{f}$ : $0.54\left(\mathrm{CH}_{2} \mathrm{Cl}_{2} / \mathrm{MeOH} 9 / 1\right) /$ silica. IR $\left(\mathrm{KBr}, \mathrm{cm}^{-1}\right): \vee(\mathrm{OH})=3438 ; \vee(\mathrm{C}=\mathrm{O})=1600 ; \vee($ enolic $\mathrm{C}=\mathrm{C})=$ 1549; ${ }^{1} \mathrm{H}-\mathrm{NMR}$ (DMSO- $\left.d_{6}\right): \delta 7.51\left(\mathrm{~s}, 1 \mathrm{H}\right.$, enol CH); $7.61\left(\mathrm{~m}, 3 \mathrm{H}, \mathrm{Ar}-\mathrm{H}_{4,3,5}\right) ; 7.66\left(\mathrm{~d}, 2 \mathrm{H}, \mathrm{Ar}-\mathrm{H}_{2,6}\right) ; 8.00$ $\left(\mathrm{t}, 1 \mathrm{H}, \mathrm{Py}-\mathrm{H}_{\beta}\right) ; 8.04\left(\mathrm{t}, 1 \mathrm{H}, \mathrm{Py}-\mathrm{H}_{\gamma}\right) ; 8.11\left(\mathrm{~d}, 1 \mathrm{H}, \mathrm{Py}-\mathrm{H}_{\delta}\right) ; 8.77(\mathrm{~d}, 1 \mathrm{H}, \mathrm{Py}-\mathrm{H} \alpha) .{ }^{13} \mathrm{C}-\mathrm{NMR}\left(\mathrm{DMSO}-d_{6}\right): \delta$ 93.6 (1C, enol C-H); 122.5 (1C, Py-C $\delta$ ); 127.7 (2C, Ar-C 2,6 ); 128.8 (1C, Ar-C $\left.\mathrm{C}_{4}\right) ; 129.4$ (2C, Ar- $\left.\mathrm{C}_{3,5}\right) ; 133.7$ (1C, Py-C $\beta$ ); 138.3 (1C, Py-C $\gamma$ ); 150.1 (1C, Py-C $\alpha$ ); 184.4 (1C, C-OH); 186.1 (1C, C=O); MS: $m / z, 226.13$ $(\mathrm{M}+\mathrm{H})^{+}$. Anal. Calcd. for $\mathrm{C}_{14} \mathrm{H}_{11} \mathrm{NO}_{2}: \mathrm{C}, 74.65 ; \mathrm{H}, 4.92 ; \mathrm{N}, 6.22$. Found: $\mathrm{C}, 74.74, \mathrm{H}, 4.89 ; \mathrm{N}, 6.25$.

(Z)-3-Hydroxy-1-(pyridin-2-yl)-3-p-tolylprop-2-en-1-one (7). Brown powder, yield 27\%, m.p.: 174-176 ${ }^{\circ} \mathrm{C} ; R_{f}: 0.75\left(\mathrm{CH}_{2} \mathrm{Cl}_{2} / \mathrm{MeOH} 6 / 4\right) /$ silica. IR $\left(\mathrm{KBr}, \mathrm{cm}^{-1}\right): v(\mathrm{OH})=3444 ; v(\mathrm{C}=\mathrm{O})=1602 ; v$ (enolic $\mathrm{C}=\mathrm{C})=1542 ;{ }^{1} \mathrm{H}-\mathrm{NMR}\left(\mathrm{DMSO}-d_{6}\right): \delta 2.49\left(\mathrm{~s}, 3 \mathrm{H}, p-\mathrm{CH}_{3}-\mathrm{Ar}\right) ; 6.78(\mathrm{~s}, 1 \mathrm{H}$, enol CH); $7.31(\mathrm{~d}, 2 \mathrm{H}$, Ar- $\left.\mathrm{H}_{3,5}\right) ; 7.46\left(\mathrm{~d}, 2 \mathrm{H}, \mathrm{Ar}-\mathrm{H}_{2,6}\right) ; 7.91\left(\mathrm{dd}, 2 \mathrm{H}, \mathrm{Py}-\mathrm{H}_{\beta, \gamma}\right) ; 8.12$ (d; 1H, Py-H $\delta$ ); 8.89 (d, 1H, Py-H $\left.\alpha\right)$. ${ }^{13} \mathrm{C}-\mathrm{NMR}$ (DMSO-d $\left.d_{6}\right): \delta 21.3\left(1 \mathrm{C}, p-\mathrm{CH}_{3}-\mathrm{Ar}\right) ; 93.8$ (1C, enol CH); $121.7\left(1 \mathrm{C}, \mathrm{Py}-\mathrm{H}_{\delta}\right) ; 126.1\left(2 \mathrm{C}, \mathrm{Ar}-\mathrm{C}_{2,6}\right)$; 128.1 (1C, Py-H $\beta$ ); 128.9 (2C, Ar-C 3,5$) ; 136.1$ (1C, Py-H $\left.{ }_{\gamma}\right) ; 149.6$ (1C, Py-H $\propto$ ); 178.1 (1C, C-OH); 186.1 $(1 \mathrm{C}, \mathrm{C}=\mathrm{O}) ; \mathrm{MS}: m / z, 240.13(\mathrm{M}+\mathrm{H})^{+}$. Anal. Calcd. for $\mathrm{C}_{15} \mathrm{H}_{13} \mathrm{NO}_{2}: \mathrm{C}, 75.30 ; \mathrm{H}, 5.48, \mathrm{~N}, 5.85$. Found: C, $75.49 ; \mathrm{H}, 5.44 ; \mathrm{N}, 5.80$.

(Z)-3-Hydroxy-3-(4-methoxyphenyl)-1-(pyridin-2-yl)prop-2-en-1-one (8). Brown clear crystal, yield 33\%, m.p.: $112-114{ }^{\circ} \mathrm{C} ; R_{f}: 0.33\left(\mathrm{CH}_{2} \mathrm{Cl}_{2} / \mathrm{MeOH} 9 / 1\right) /$ silica. IR $\left(\mathrm{KBr}, \mathrm{cm}^{-1}\right): v(\mathrm{OH})=3444 ; v(\mathrm{C}=\mathrm{O})=$ 1599; v (enolic $\mathrm{C}=\mathrm{C})=1549 ;{ }^{1} \mathrm{H}-\mathrm{NMR}\left(\mathrm{DMSO}-\mathrm{d}_{6}\right): \delta 3.83\left(\mathrm{~s}, 3 \mathrm{H}, \mathrm{O}-\mathrm{CH}_{3}\right) ; 7.08\left(\mathrm{~d}, 2 \mathrm{H}, \mathrm{Ar}-\mathrm{H}_{3,5}\right) ; 7.50$ $(\mathrm{s}, 1 \mathrm{H}$, enol, $\mathrm{C} \underline{\mathrm{H}}) ; 7.62\left(\mathrm{t}, 1 \mathrm{H}, \mathrm{Py}-\mathrm{H}_{\delta}\right) ; 7.97\left(\mathrm{t}, 1 \mathrm{H}, \mathrm{Py}-\mathrm{H}_{\beta}\right) 8.04\left(\mathrm{~d}, 2 \mathrm{H}, \mathrm{Ar}-\mathrm{H}_{2,6}\right) ; 8.08\left(\mathrm{t}, 1 \mathrm{H}, \mathrm{Py}^{-} \mathrm{H}_{\gamma}\right)$; $8.76(\mathrm{~d}, 1 \mathrm{H}, \mathrm{Py}-\mathrm{H} \alpha) .{ }^{13} \mathrm{C}-\mathrm{NMR}\left(\mathrm{DMSO}_{6}\right)$ : $\delta 56.1\left(1 \mathrm{C}, \mathrm{OCH}_{3}\right) ; 92.8(1 \mathrm{C}$, enol, $\mathrm{CH}) ; 114.5\left(2 \mathrm{C}, \mathrm{Ar}-\mathrm{C}_{3,5}\right)$; 
122.2 (1C, Py-C $\delta$ ); 127.5 (2C, Ar-C 2,6 ); 138.2 (1C, Py-C C ); 150.1 (1C, Py-C $\alpha) ; 182.1$ (1C, C-OH); 186.6 $(1 \mathrm{C}, \mathrm{C}=\mathrm{O})$; MS: $m / z, 256.08(\mathrm{M}+\mathrm{H})^{+}$. Anal. Calcd. for $\mathrm{C}_{15} \mathrm{H}_{13} \mathrm{NO}_{3}: \mathrm{C}, 70.58 ; \mathrm{H}, 5.13 ; \mathrm{N}, 5.49$. Found: C, 70.76; H, 5.20, N, 5.59.

(Z)-1-(Furan-2-yl)-3-hydroxybut-2-en-1-one (9). Red hygroscopic; yield: 39\%; $R_{f}$ : $0.91\left(\mathrm{CH}_{2} \mathrm{Cl}_{2} / \mathrm{MeOH}\right.$ $8 / 2) /$ silica. IR $\left(\mathrm{KBr}, \mathrm{cm}^{-1}\right): \vee(\mathrm{OH})=3434 \mathrm{~cm}^{-1} ; \vee(\mathrm{C}=\mathrm{O})=1620 \mathrm{~cm}^{-1} ; \vee($ enolic $\mathrm{C}=\mathrm{C})=1468$; ${ }^{1} \mathrm{H}-\mathrm{NMR}\left(\mathrm{CDCl}_{3}\right)$ : $\delta 2.13$ (s, 3H, - $\left.\mathrm{CH}_{3}\right) ; 6.06\left(\mathrm{~s}, 1 \mathrm{H}\right.$, enol, C-H); $6.52\left(\mathrm{~m}, 1 \mathrm{H}, \mathrm{Fu}-\mathrm{H}_{\beta}\right) ; 7.14(\mathrm{~d}, 1 \mathrm{H}$, Fu- $\left.\mathrm{H}_{\gamma}\right) ; 7.55(\mathrm{~m}, 1 \mathrm{H}, \mathrm{Fu}-\mathrm{H} \alpha) ;{ }^{13} \mathrm{C}-\mathrm{NMR}\left(\mathrm{CDCl}_{3}\right): \delta 24.4$ (1C, $\left.\mathrm{CH}_{3}-\mathrm{C}=\mathrm{O}\right) ; 96.0$ (1C, C-H, enol); 112.4 (1C, Fu-C $\gamma$ ); 115.5 (1C, Fu-C $\left.{ }_{\beta}\right) ; 145.9$ (1C, Fu-C $\left.\alpha\right) ; 150.0$ (1C, Fu-C $\delta$ ); 176.1 (1C, C=O), 19.5 (1C, C-OH); MS: $m / z, 153.10(\mathrm{M}+\mathrm{H})^{+}$. Anal. Calcd. for $\mathrm{C}_{8} \mathrm{H}_{8} \mathrm{O}_{3}$ : C, 63.15; H, 5.30. Found: $\mathrm{C}, 63.25, \mathrm{H}, 5.38$.

(Z)-1-(Furan-2-yl)-3-hydroxy-3-phenylprop-2-en-one (10). Red powder; yield 42\%; m.p. $64-66{ }^{\circ} \mathrm{C}$; $R_{f}$ : $0.61\left(\mathrm{CH}_{2} \mathrm{Cl}_{2}\right.$ /silica). IR $\left(\mathrm{KBr}, \mathrm{cm}^{-1}\right): \vee(\mathrm{OH})=3431 ; \vee(\mathrm{C}=\mathrm{O})=1621 ; \vee($ enolic $\mathrm{C}=\mathrm{C})=1531$; ${ }^{1} \mathrm{H}-\mathrm{NMR}\left(\mathrm{DMSO}-d_{6}\right): \delta 6.72\left(\mathrm{~d}, 1 \mathrm{H}, \mathrm{Fu}-\mathrm{H}_{\gamma}\right) ; 7.01\left(\mathrm{~s}, 1 \mathrm{H}\right.$, enol, C-H); $7.52\left(\mathrm{~d}, 2 \mathrm{H}, \mathrm{Ar}-\mathrm{H}_{3,5}\right) ; 7.58(\mathrm{t}, 1 \mathrm{H}$, $\left.\mathrm{Ar}-\mathrm{H}_{4}\right) ; 7.64\left(\mathrm{~d}, 2 \mathrm{H}, \mathrm{Ar}-\mathrm{H}_{2,6}\right) ; 8.00\left(\mathrm{t}, 1 \mathrm{H}, \mathrm{Fu}-\mathrm{H}_{\beta}\right) ; 8.04$ (d, $\left.1 \mathrm{H}, \mathrm{Fu}-\mathrm{H} \alpha\right) ;{ }^{13} \mathrm{C}-\mathrm{NMR}\left(\mathrm{DMSO}-d_{6}\right): \delta 93.3$ (1C, C-H, enol); 113.5 (1C, Fu-C $)$; $120.2\left(1 \mathrm{C}, \mathrm{Fu}-\mathrm{C}_{\beta}\right) ; 127.3\left(2 \mathrm{C}, \mathrm{Ar}-\mathrm{C}_{3,5}\right) ; 129.2\left(3 \mathrm{C}, \mathrm{Ar}-\mathrm{C}_{2,4,6}\right) ; 148.7$ (1C, Fu-C $\alpha) ; 178.5(1 \mathrm{C}, \mathrm{C}=\mathrm{O}) ; 180.8(1 \mathrm{C}, \mathrm{C}-\mathrm{OH}) ; \mathrm{MS}: m / z, 215.12(\mathrm{M}+\mathrm{H})^{+}$.

\subsection{Anticancer Assays}

Prepared compounds were screened against breast cancer (MDA-MB241) human cell lines using normoxic conditions [20]. Tests were performed in Angiogenesis and Cancer Research Lab, Institute of Experimental and Clinical Research (UCL, Brussels, Belgium).

\subsection{Antibacterial and Antifungal Tests}

The in vitro antifungal activities were tested by the agar diffusion technique [21] using fungal strains (Fusarium oxysporum f.sp albedinis FAO).

The results were compared with positive controls (benomyl and thiophanate-methyl).The in vitro antibacterial activities were tested using bacterial strains (Echerichia coli, Bacillus subtilis, and Micrococcus luteus).

\section{Conclusions}

In summary, we have described the first synthesis of novel $\beta$-keto-enols embedded with heterocyclic moieties and the evaluation of their in vitro anticancer and antifungal activities. Most of the compounds showed modest antiproliferative activity against breast cancer (MDA-MB241) human cell lines. Among the synthesized products, compounds 1, 5, and 9 successfully showed the most potent antifungal activity with $\mathrm{IC}_{50}$ values in the range of $0.055-0.092 \mu \mathrm{M}$ as compared with positive controls.

Acknowledgments: The authors would like to extend their sincere appreciation to the Deanship of Scientific Research at King Saud University for its funding this Research group No (RG-007-2015).

Author Contributions: S.R. and S.T. carried out the experimental work and cooperated in the preparation of the manuscript. O.R. provided much of the starting chemicals products. O.F. performed the anticancer assays. M.B. and R.B. performed the antifungal assays. Y.N.M. interpreted the results and edited the English language.

Conflicts of Interest: The authors declare no conflict of interest.

\section{References}

1. Pommier, Y.; Johnson, A.A.; Marchand, C. Integrase inhibitors to treat HIV/AIDS. Nat. Rev. Drug Discov. 2005, 4, 236-248. [CrossRef] [PubMed]

2. Egbertson, S.S.; Anthony, N.J.; Summa, V. HIV integrase inhibitors: From diketo acids to heterocyclic templates: History of HIV integrase medicinal chemistry at Merck West Point and Merck Rome (IRBM) leading to discovery of raltegravir. In Pharmaceutical \& Medicinal Chemistry; Neamati, N., Ed.; John Wiley \& Sons: Hoboken, NJ, USA, 2011; Chapter 14; pp. 197-230. 
3. Goldgur, Y.; Craigir, R.; Cohen, G.H.; Fujiwara, T.; Yoshinaga, T.; Fujishita, T.; Sugimoto, H.; Endo, T.; Murai, H.; Davies, D.R. Structure of the HIV-1 integrase catalytic domain complexed with an inhibitor: A platform for antiviral drug design. Proc. Natl. Acad. Sci. USA 1999, 96, 13040-13043. [CrossRef] [PubMed]

4. Hazuda, D.J.; Felock, P.; Witmer, M.; Wolfe, A.; Stillmock, K.; Grobler, J.A.; Espeseth, A.; Gabryelski, L.; Schleif, W.; Blau, C.; et al. Inhibitors of strand transfer that prevent integration and inhibit HIV-1 replication in cells. Science 2000, 287, 646-650. [CrossRef] [PubMed]

5. Pluymers, W.; Pais, G.; Maele, B.V.; Pannecouque, C.; Fikkert, V.; Burke, J.T.R.; de Clercq, E.; Witvrouw, M.; Neamati, N.; Debyser, Z. Inhibition of human immunodeficiency virus type 1 integration by diketo derivatives. Antimicrob. Agents Chemother. 2002, 46, 3292-3297. [CrossRef] [PubMed]

6. Ishikawa, Y.; Fujii, S. Binding Mode prediction and inhibitor design of anti-influenza virus diketo acids targeting metalloenzyme RNA polymerase by molecular docking. Bioinformation 2011, 6, 221-225. [CrossRef] [PubMed]

7. Kohyama, A.; Yamakoshi, H.; Hongo, S.; Kanoh, N.; Shibata, H.; Iwabuchi, Y. Structure-activity relationships of the antitumor C5-curcuminoid GO-Y030. Molecules 2015, 20, 15374-15391. [CrossRef] [PubMed]

8. Anand, P.; Thomas, S.G.; Kunnumakkara, A.B.; Sundaram, C.; Harikumar, K.B.; Sung, B.; Tharakan, S.T.; Misra, K.; Priyadarsini, I.K.; Rajasekharan, K.N.; et al. Biological activities of curcumin and its analogues (Congeners) made by man and Mother Nature. Biochem. Pharmacol. 2008, 76, 1590-1611. [CrossRef] [PubMed]

9. Minassi, A.; Sanchez-Duffhues, G.; Collado, J.A.; Munoz, E.; Appendino, G. Dissecting the pharmacophore of curcumin. Which structural element is critical for which action? J. Nat. Prod. 2013, 76, 1105-1112. [CrossRef] [PubMed]

10. Adams, B.K.; Cai, J.; Armstrong, J.; Herold, M.; Lu, Y.J.; Sun, A.; Snyder, J.P.; Liotta, D.C.; Jones, D.P.; Shoji, M. EF24, a novel synthetic curcumin analog, induces apoptosis in cancer cells via a redox-dependent mechanism. Anti-Cancer Drug. 2005, 16, 263-275. [CrossRef]

11. Tan, K.L.; Ali, A.; Du, Y.; Fu, H.; Jin, H.X.; Chin, T.M.; Khan, M.; Go, M.L. Synthesis and evaluation of bisbenzylidenedioxotetrahydrothiopranones as activators of endoplasmic reticulum (ER) stress signaling pathways and apoptotic cell death in acute promyelocytic leukemic cells. J. Med. Chem. 2014, 57, 5904-5918. [CrossRef] [PubMed]

12. Liang, G.; Shao, L.; Wang, Y.; Zhao, C.; Chu, Y.; Xiao, J.; Zhao, Y.; Li, X.; Yang, S. Exploration and synthesis of curcumin analogues with improved structural stability both in vitro and in vivo as cytotoxic agents. Bioorg. Med. Chem. 2009, 17, 2623-2631. [CrossRef] [PubMed]

13. Liang, G.; Li, X.; Chen, L.; Yang, S.; Wu, X.; Studer, E.; Gurley, E.; Hylemon, P.B.; Ye, F.; Li, Y.; et al. Synthesis and anti-inflammatory activities of mono-carbonyl analogues of curcumin. Bioorg. Med. Chem. Lett. 2008, 18, 1525-1529. [CrossRef] [PubMed]

14. Luo, Z.-G.; Zhao, Y.; Ma, C.; Xu, X.-M.; Zhang, X.-M.; Huang, N.-Y.; He, H.-Q. Synthesis and anti-integrase evaluation of novel calix[4]arene derivatives containing the triazolyl 1,3-diketo moiety. Chin. Chem. Lett. 2014, 25, 737-740. [CrossRef]

15. Song, W.-H.; Liu, M.-M.; Zhong, D.-W.; Zhu, Y.-L.; Bosscher, M.; Ye, D.-Y.; Yuan, Z.-H.; Zhou, L. Tetrazole and triazole as bioisosteres of carboxylic acid: Discovery of diketo tetrazoles and diketo triazoles as anti-HCV agents. Bioorg. Med. Chem. Lett. 2013, 23, 4528-4531. [CrossRef] [PubMed]

16. Patel, K.; Karthikeyan, C.; Solomon, V.R.; Moorthy, N.S.H.N.; Lee, H.; Sahu, K.; Deora, G.S.; Trivedi, P. Synthesis of some coumarinyl chalcones and their antiproliferative activity against breast cancer cell lines. Lett. Drug Des. Discov. 2011, 8, 308-311. [CrossRef]

17. Radi, S.; Tighadouini, S.; Ben Hadda, T.; Akkurt, M.; Özdemir, N.; Sirajuddin, M.; Mabkhot, Y.N. Crystal structure of (2Z)-3-hydroxy-1-(1,5-dimethyl-1H-pyrazol-3-yl)but-2-en-1-one, $\mathrm{C}_{9} \mathrm{H}_{12} \mathrm{~N}_{2} \mathrm{O}_{2}$. Z. Kristallogr. 2015. submitted.

18. Riahi, A.; Wurster, M.; Lalk, M.; Lindequist, U.; Langer, P. Synthesis and antimicrobial activity of 4-hydroxy-4-(pyridyl)alk-3-en-2-ones. Bioorg. Med. Chem. 2009, 17, 4323-4326. [CrossRef] [PubMed]

19. Hansen, P.E.; Borisov, E.V.; Lindon, J.C. Determination of the tautomeric equilibria of pyridoyl benzoyl $\beta$-diketones in the liquid and solid state through the use of deuterium isotope effects on ${ }^{1} \mathrm{H}$ and ${ }^{13} \mathrm{C}$-NMR chemical shifts and spin coupling constants. Spectrochim. Acta A Mol. Spectrosc. 2015, 136, 107-112. [CrossRef] [PubMed] 
20. Defresne, F.; Bouzin, C.; Guilbaud, C.; Dieu, M.; Delaive, E.; Michiels, C.; Raes, M.; Feron, O. PP 82 Pleiotropic influences of radio- and chemotherapy on auto-antibodies warrant caution for their use as biomarkers of tumor response: The anti-GRP78 paradigmatic example. Eur. J. Cancer 2011, 47, S16-S16. [CrossRef]

21. Carrod, L.P.; Grady, F.D. Antibiotics and Chemotherapy, 3rd ed.; Churchill Livingstone: Edinburgh, UK, 1972; p. 477.

Sample Availability: Samples of the compounds are available from the authors.

(C) 2015 by the authors; licensee MDPI, Basel, Switzerland. This article is an open access article distributed under the terms and conditions of the Creative Commons by Attribution (CC-BY) license (http://creativecommons.org/licenses/by/4.0/). 